\title{
Aspectos históricos da implantação de um serviço de atendimento pré-hospitalar
}

\author{
Historical aspects of the implantation of a prehospital care service
}

\section{Aspectos históricos de la implantación de un servicio de atención prehospitalaria}

Elisângelo Aparecido Costa da Silva', Anaclara Ferreira Veiga Tipple", J oaquim Tomé de Souza'I', Virginia Visconde Brasil'V

' Enfermeiro. Mestre em Enfermagem. Docente da Universidade Paulista. Goiânia, GO, Brasil. E-mail: elisangelo@hotmail.com.

"Enfermeira. Doutora em Enfermagem. Professor Associado, Faculdade de Enfermagem (FEN), Universidade Federal de Goiás (UFG). Goiânia, GO, Brasil.

E-mail: anaclara@fen.ufg.br.

III Médico. Doutor em Ciência. Goiânia, Go, Brasil. E-mail: tome@superiq.com.br.

IV Enfermeira. Doutora em Enfermagem. Professor Associado, FEN, UFG. Goiânia, GO, Brasil. E-mail: virqinia@fen.ufg.br.

\section{RESUMO}

O serviço de atendimento pré-hospitalar (APH) desempenha importante papel na saúde pública, uma vez que as doenças cardiovasculares e os eventos relacionados às causas externas como situações emergenciais são observadas cada vez mais na população em geral. A reunião de informações históricas permite ao leitor se inteirar rapidamente de como ocorreu a criação e estruturação dos serviços de atendimento pré-hospitalar. Os objetivos foram descrever os aspectos históricos da estruturação do serviço de atendimento pré-hospitalar e sua implantação e evolução no estado de Goiás. Trata-se de uma atualização que procura resgatar dados históricos relacionados ao APH em Goiás. Observa-se que, no Brasil, a formação das equipes de profissionais e a forma da abordagem no atendimento às vítimas foram baseadas nos modelos americano e francês de APH. São políticas públicas de saúde ligadas à atenção às urgências ainda novas e caminham para o aprimoramento. Como profissionais da área da saúde e/ou do APH, somos co-responsáveis pelo alcance do objetivo primordial que é a excelência no atendimento.

Descritores: Busca e resgate; Serviços médicos de emergência; Assistência pré-hospitalar.

\section{ABSTRACT}

The prehospital care service plays important role in public health, as cardiovascular disease and events related to external causes such as emergency situations are increasing in the general population. The reunion of historical information enables the reader to know how the creation and structuring of prehospital care services happened. The objectives were to describe the historical aspects of the structuring of the prehospital care service, its implementation and evolution in Goiás state. This update seeks to rescue historical data related to prehospital care services in Goiás. In Brazil the training of professionals and the way to assist the victims were based on the American and French models of prehospital care. They are public health policies related to emergency care that are new and need to go further and to be improved. As health professionals we are co-responsible for improving the quality of care.

Descriptors: Search and rescue; Emergency medical services; Prehospital care.

\section{RESUMEN}

El servicio de atención prehospitalaria desempeña papel importante en la salud pública, una vez que las enfermedades cardiovasculares y eventos relacionados con las causas externas, como las situaciones de emergencia, se ven cada vez más en la población general. La histórica reunión de información permite que el lector obtenga informaciones al respecto de la creación y estructuración de los servicios de atención prehospitalaria. Los objetivos fueron describir los aspectos históricos de la estructuración del servicio de atención prehospitalaria y de su aplicación y la evolución en el estado de Goiás, a través de una actualización que busca rescatar los datos históricos relacionados con el servicio de atención prehospitalaria en el estado de Goiás. Se observa que en el Brasil la formación de equipos de profesionales y la forma de cuidado de las víctimas se basaron en los modelos americano y francés de atención prehospitalaria. Las políticas de salud pública relacionadas con la atención de emergencias son nuevas e caminan para el aprimoramiento. Como profesionales de la salud somos coresponsables par la mejora de la calidad de la atención.

Descriptores: Búsqueda y rescate; Servicios médicos de urgencia; Atención Prehospitalaria. 


\section{NTRODUÇÃO}

$O$ atendimento nas áreas de urgência e emergência tem crescido e se tornado cada vez mais expressivo na sociedade brasileira e mundial. O aumento dos casos de acidentes e violência tem forte impacto sobre o Sistema Único de Saúde (SUS) do país e o conjunto da sociedade. $\mathrm{Na}$ assistência, este impacto pode ser medido diretamente pelo aumento dos gastos com internações hospitalares, assistência em Unidade de Terapia Intensiva (UTI) e tempo de hospitalização. Na questão social, pode ser verificado pelo aumento de $30 \%$ no índice de Anos Potenciais de Vida Perdidos (APVP) devido a acidentes e violências nos últimos $\operatorname{anos}^{(1)}$.

No Brasil as internações por causas externas no período de janeiro de 2006 a janeiro de 2007 entre indivíduos de 20 a 49 anos somaram 416.253 casos. Destes, 37.100 ocorreram na região Centro-Oeste, sendo 14.666 em todo Estado de Goiás e 9.537 casos na capital goiana $^{(2)}$

As afecções por causas externas, a violência urbana e as doenças cardiovasculares, respiratórias e metabólicas dentre outras, são situações críticas para uma pessoa, e determinam a necessidade de atendimento imediato emergencial e definitivo. Ao longo do tempo percebeu-se que vidas poderiam ser salvas se fossem rapidamente atendidas por pessoas treinadas e qualificadas, ainda no ambiente fora dos hospitais, denominado atendimento préhospitalar (APH), e transportadas a um local onde pudessem receber atendimento com suporte mais específico para cada caso $^{(3)}$. Tem sido enfatizado como fator crítico para a sobrevivência da vítima (retorno às atividades habituais sem sequelas), o tempo gasto até que o tratamento definitivo seja instalado ${ }^{(4)}$. O período entre o reconhecimento e resposta quando o indivíduo está em risco, fará diferença entre "vida e morte" e entre "sobrevivência intacta e sequelas"(5).

O interesse para o estudo desse tema ocorreu no contexto da realização da dissertação de mestrado abordando o risco biológico dos profissionais do atendimento pré-hospitalar ${ }^{(6)}$. Foi possível reconhecer a riqueza dos aspectos históricos que envolveram o desenvolvimento do cuidado inicial às vítimas e perceber a influência dos fatos que determinaram a atual situação do atendimento pré-hospitalar. Por outro lado, observou-se o reduzido número de registros na literatura nacional e internacional, e principalmente os fatos relacionados ao estado de Goiás.

A reunião de informações históricas permite ao leitor se inteirar rapidamente de como ocorreu a criação e estruturação dos serviços de atendimento pré-hospitalar, influenciados pelos modelos mundiais na sua implantação. Assim, os objetivos deste estudo foram descrever os aspectos históricos da estruturação do serviço de atendimento pré-hospitalar e sua implantação e evolução no estado de Goiás.

\section{Aspectos históricos sobre o Atendimento Pré- Hospitalar}

Condições de risco para a vida das pessoas sempre existiram e junto com esses riscos, pelo instinto de preservação da vida, o ser humano teve a preocupação de buscar o mais precoce possível, um suporte para manter as condições vitais do indivíduo.

Embora não descrito com a finalidade de explicitar um atendimento a uma vítima de violência, um dos primeiros registros identificados está na Bíblia, no livro de Lucas, capítulo 10, versículos 30 a 34 , onde se lê: “... certo homem descia de Jerusalém para Jericó e veio a cair em mãos de salteadores, os quais, depois de tudo Ihe roubarem e lhe causarem muitos ferimentos, retiraram-se, deixando-o semimorto. Casualmente, descia um sacerdote por aquele mesmo caminho e, vendo-o, passou de largo. Semelhantemente, um levita descia por aquele lugar e, vendo-o, também passou de largo. Certo samaritano, que seguia o seu caminho, passou-lhe perto e, vendo-o, compadeceu-se dele. E, chegando-se, pensou-lhe os ferimentos, aplicando-Ihe óleo e vinho; e, colocando-o sobre o seu próprio animal, levou-o para uma hospedaria e tratou dele" ${ }^{\prime(7)}$

Falar em preservação da vida ao longo dos séculos implica lembrar como foi possível ressuscitar indivíduos "aparentemente mortos". Isso só foi considerado possibilidade científica, a partir do século XVIII. Até então, há registros de sucesso, mas envoltos em misticismo e crenças religiosas. As manobras de ressuscitação foram desenvolvidas e tornaram-se realidade após os anos $1960^{(8)}$.

O socorro sistematizado emergencial prestado às vítimas de situações críticas teve suas bases alicerçadas durante a guerra civil americana, onde eram perdidas muitas vidas, principalmente de soldados, por falta de atendimento imediato. Foi identificada a necessidade de providências para agilizar o atendimento às vítimas ainda no campo de batalha. Alguns conceitos como segurança da cena (evitar tornar-se mais uma vítima, evitar a ocorrência de novas vítimas), exame primário (tratamento das lesões em risco de vida, evitar mais dano) e a própria questão do transporte rápido (para o local de tratamento definitivo), são oriundos dessa época ${ }^{(9)}$.

Tecnicamente, o marco da criação da ambulância projetada deve-se ao médico Dominique J ean Larrey (17661842), considerado "Pai da Medicina Militar". Como cirurgião do exército napoleônico, identificou a necessidade de resgatar os feridos não apenas após o término do conflito, mas ainda durante a batalha. Larrey, necessitando estabelecer atendimento imediato, projetou Unidades de Transporte de feridos, que batizou como "ambulâncias voadoras", pois tinham como características serem leves e velozes (Figura 1). O aumento da velocidade deu-se pelo uso, inicialmente, de dois cavalos lado a lado e posteriormente perfilados, bem como madeira leve, rodas pequenas e teto arredondado para evitar retenção de água na madeira durante a chuva ${ }^{(10)}$. 


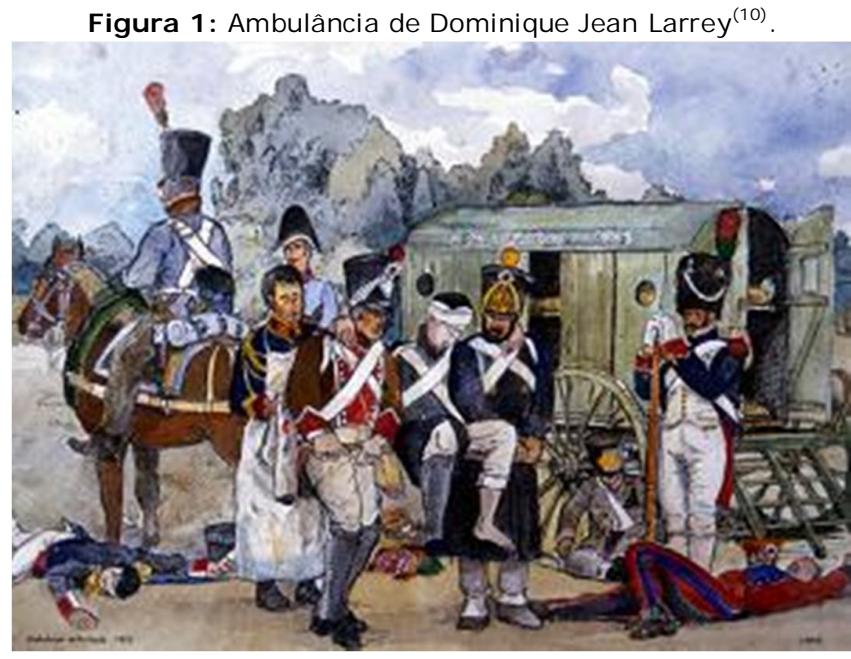

Para conforto do paciente havia ainda duas perfurações laterais para ventilação, acondicionamento de maca, cobertores para aquecimento e guarda de instrumentos ${ }^{(11)}$. As ambulâncias passaram então a buscar feridos imediatamente, tendo sido obtida importante redução na mortalidade.

Com o advento da era industrial, no final do século XIX, surgiram os motores a combustão, imediatamente incorporados a modelos confortáveis e seguros. A equipe já era composta por condutor, pessoal de enfermagem e eventualmente, o médico. Em 1900, as unidades estavam motorizadas e havia equipes específicas, já da recente criada Cruz Vermelha (Figura 2). Hoje, a evolução tecnológica permitiu o avanço das unidades móveis, que conta com equipe treinada, equipamentos microprocessados, serviço de comunicação, velocidade rápida, climatização, arsenal terapêutico e normatizações que regem 0 atendimento ${ }^{(10)}$

Figura 2: Ambulância de 1899 do Corpo de Bombeiros Militar do Rio de Janeiro, Brasil ${ }^{(10)}$.

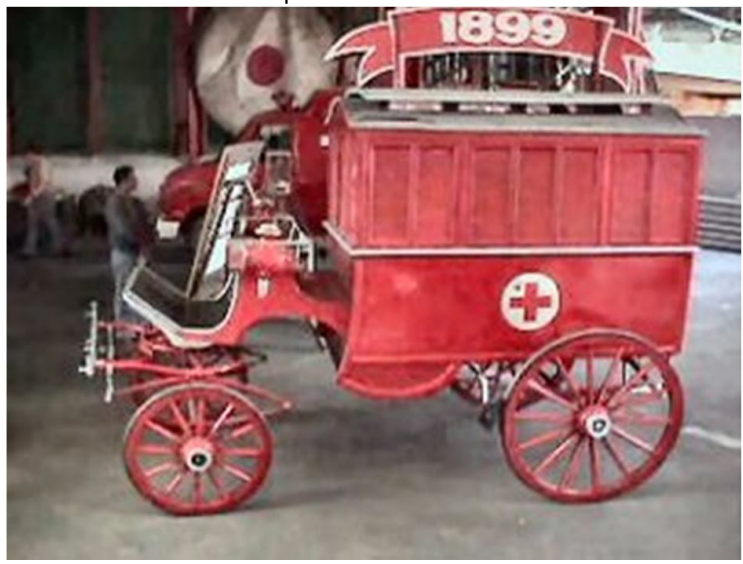

As guerras do Vietnã e Coréia, comparadas com a Segunda Guerra Mundial, demonstraram que a rapidez na remoção dos feridos dos campos de batalha, associada a medidas de estabilização do paciente durante o transporte reduzia significativamente a mortalidade. Cada 30 minutos de retardo na remoção aumentavam a mortalidade em três vezes, e os cuidados elementares reduziam em 20\% a mortalidade dos feridos ${ }^{(11)}$.

Técnicas e protocolos foram aprimorados à medida que novas situações de emergência apareciam e o maior destaque dado às situações de guerra e militaria. Uma dessas contribuições foi, por exemplo, a introdução do uso de helicópteros no resgate de vítimas, a partir de 1970. Observa-se então a incorporação militar nos serviços de emergência, como o Corpo de Bombeiros.

No Brasil o surgimento dos serviços de emergência pré-hospitalar foi influenciado pelos modelos americano e francês. A França destaca-se no cenário mundial pelo seu serviço APH, por construir um modelo bastante eficiente, com órgãos permanentes e temporários, obedecendo a uma orientação centralizada, amparada por legislação pertinente, bem como recursos humanos e materiais de acordo com as necessidades levantadas por planejamento ${ }^{(10)}$.

O modelo francês é centralizado numa rede de comunicações e baseado na regulação médica. Todas as chamadas são avaliadas por um médico, que define a resposta mais eficiente, maximizando os recursos disponíveis. Essa experiência vem sendo avaliada há anos, mostrando ser importante instrumento para as ações em saúde, uma vez que permite o conhecimento das necessidades reais da vítima e dos recursos disponíveis à prestação da assistência, dentre outras informações, possibilitando o gerenciamento da demanda ${ }^{(12)}$.

Nos Estados Unidos, o APH começou a ser mais bem organizado em 1966, quando o governo americano determinou que a segurança rodoviária desenvolvesse um 
sistema eficiente de atendimento, para diminuir as estatísticas de morte por situações de urgência e emergência(10,12). Desta forma, em 1968 foi criado um número telefônico único (911), para centralizar os chamados de emergência. A partir daí, as emergências médicas eram transmitidas aos profissionais da área que se encarregavam de enviar o melhor recurso(13).

O serviço de APH no Brasil tem um histórico ligado à instituição militar. O primeiro registro pode ser observado em 1899, quando o Corpo de Bombeiros do Rio de Janeiro (CBMRJ), capital do país na época, colocou em ação a primeira ambulância de tração animal, para realizar atendimento no ambiente fora do hospital ${ }^{(14-15)}$.

A partir de 1900, com o surgimento dos primeiros modelos motorizados, principalmente após as experiências das I e II Grandes Guerras, as ambulâncias foram aprimoradas e melhor adequadas ao serviço, primeiramente pelas equipes especializadas como da Cruz Vermelha Internacional e depois assimiladas pelos serviços do Corpo de Bombeiros brasileiro ${ }^{(10)}$.

$\mathrm{Na}$ década de 1960 uma segunda tentativa de implantar o serviço de atendimento APH ocorreu no Brasil, a partir de uma política nacional, com o título de Serviço de Atendimento Médico Domiciliar de Urgência - SAMDU, e tinha por finalidade o atendimento nas residências com a presença de um médico ou acadêmico de medicina na ambulância coordenando a equipe.

No âmbito federal, destaca-se a proposta da Política Nacional de Atenção às Urgências e o Serviço de Atendimento Móvel de Urgência (SAMU) do Sistema Único de Saúde ${ }^{(1)}$. Para o desenvolvimento dessa política, o estado de São Paulo teve uma participação importante, iniciando o projeto de Resgate desenvolvido em conjunto com a Secretaria Estadual de Saúde e Secretaria de Segurança Pública (Resolução no 42 de 22/05/89). Era oferecido serviço de Atendimento Pré-Hospitalar (APH) com Unidades de Resgate (UR) tripuladas por bombeiros socorristas e Unidades de Suporte Avançado (USA) tripuladas por médicos e enfermeiros do SAMU ${ }^{(3)}$.

O Sistema Integrado de Atendimento ao Trauma e Emergência (SIATE) é outro modelo de APH, proposto por uma política federal com início de implantação em 1990, sendo Curitiba-PR o município que se destacou nesse serviço, seguido de outras cidades como Goiânia-GO, em 2000. Com o fim do SAMDU, muitos serviços de emergência, sobretudo nas capitais, passaram a enviar ambulâncias, na maioria das vezes, apenas com motorista e padioleiro, para remoção de pacientes em situação crítica, que estivessem em domicílio ou via pública, sem haver preparo específico destes profissionais ${ }^{(16)}$.

O Rio de Janeiro foi pioneiro nesse serviço, quando em 1975, com a Lei 6.299, o município ficou com a responsabilidade do atendimento às urgências, que contava com veículos e motoristas para o transporte rápido. Já em 1986, surgiu nesta cidade o Grupo de Socorro e Emergência (GSE) do Corpo de Bombeiros Militar do Rio de Janeiro, incorporando médicos ao quadro de socorristas e implementando viaturas de suporte avançado de vida com recursos materiais específicos a este fim.

Atualmente, os serviços de APH estão integrados dentro de uma mesma lógica: medidas preventivas, redes de atendimento pré-hospitalar, serviços assistenciais hospitalares hierarquizados e centros de reabilitação ${ }^{(1,12-13)}$.

A Criação do Corpo de Bombeiros Militar do Estado de Goiás (CBMGO) teve início em cinco de novembro de 1957 , com o deslocamento de 11 militares para o Estado de Minas Gerais, com a finalidade de frequentar um curso de bombeiros com duração de oito meses ${ }^{(17)}$.

Em 17 de dezembro de 1958 foi editada a Lei Estadual no. 2.400 que criava uma Companhia de Bombeiros, transferindo-se posteriormente para uma edificação próxima ao Lago das Rosas, em Goiânia/GO. Naquela época o "trem de socorro" era composto de um Auto Bomba Tanque (ABT), tipo Thames 2.000 e uma viatura Pirsch - Auto Pó Químico, ambas doadas pelo então Governador do Rio Janeiro, Carlos Lacerda ${ }^{(17)}$.

Pela Lei no. 5442, de 10 de novembro de 1964, a Companhia de Bombeiros passou a denominar-se Corpo de Bombeiros, com o efetivo de Batalhão. Em 14 de novembro de 1967, o Corpo de Bombeiros recebeu a estrutura de Batalhão. Com base na Lei no. 8125, de 18 de dezembro de 1976, Art. 2으, 3으, 9으 e 11으, combinado com o Decreto ㄲo. 1936, de 27 de agosto de 1981, foi publicada a Portaria no 04/81 criando no Corpo de Bombeiros os seguintes órgãos: Comando do Corpo de Bombeiros (CCB); Grupamento de Incêndio ( $1 \circ \mathrm{G} \mathrm{GI}) ;$ Seção de Combate a Incêndio (1aㅡ $\mathrm{SCl}$ ), com sede no Aeroporto Santa Genoveva; Seção de Combate a Incêndio ( $\left.2^{a} \mathrm{SCl}\right)$, com sede no bairro de Campinas; Seção de Combate a Incêndio ( 3 a $\mathrm{SCl}$ ) com sede na Cidade de Anápolis/GO; Seção de Combate a Incêndio (4ạ SCI), na cidade de Itumbiara/GO; Seção Contra Incêndio - SCI, na Cidade de Rio Verde/GO ${ }^{(17)}$.

Pela Constituição Estadual, promulgada em 05 de outubro de 1989, o Corpo de Bombeiros passou a constituirse uma corporação independente e autônoma, com as seguintes missões: a execução de atividades de defesa civil; a prevenção e o combate a incêndios e a situações de pânico, assim como ações de busca e salvamento de pessoas e bens; o desenvolvimento de atividades educativas relacionadas com a defesa civil e a prevenção de incêndio e pânico; a análise de projetos e inspeção de instalações preventivas de proteção contra incêndio e pânico nas edificações, para fins de funcionamento ${ }^{(17)}$.

Dentre essas unidades destaca-se em outubro de 1989, a implantação do Quartel do Comando Geral, na Avenida Anhanguera no. 6750 no Setor Aeroporto, onde a partir de 04 de fevereiro de 2000, com a mudança do Comando Geral do CBMGO para o Palácio da Segurança Pública, situado na Avenida Anhanguera, no. 7364 - Setor Aeroviário se transformou no Quartel Lago das Rosas (sede da Diretoria de Saúde, Gerência de Apoio Logístico, Grupo de Resgate Pré-hospitalar (GRPH) e Centro de Operações do Corpo de Bombeiro ${ }^{(17)}$.

Inicialmente, as atividades realizadas pelas ambulâncias do GRPH eram transporte de vítimas até os estabelecimentos de saúde de referência, com assistência de primeiros socorros pelos bombeiros militares socorristas. Esses profissionais tinham formação técnica na área de suporte básico às emergências, como suporte ventilatório, imobilizações e manutenção da estabilidade dos sinais vitais. Em 2000, a Secretaria Estadual de Saúde do Estado de Goiás criou o Sistema Integrado de Atendimento ao Trauma e Emergências (SIATE), enviando recursos materiais e humanos para agir em convênio com o Corpo de Bombeiros, oferecendo à população do estado um serviço de APH também de Suporte Avançado. Contratou enfermeiros e médicos para as ambulâncias dos bombeiros e adquiriu equipamentos necessários ao suporte avançado.

Atualmente existem dois serviços de atendimento de urgência e emergência pré-hospitalar móvel público em 
Goiânia-GO: o Sistema Integrado de Atendimento ao Trauma e Emergência (órgão da Secretaria Estadual de Saúde que atua em convênio com a Secretaria de Segurança Pública e Corpo de Bombeiros Militar do Estado de Goiás) e o Serviço de Atendimento Móvel de Urgência (SAMU) na esfera municipal.

Esse atendimento é feito 24 horas por dia em qualquer lugar (residências, locais de trabalho e vias públicas), depois de uma chamada gratuita aos telefones 192 e/ou 193. A ligação é atendida por técnicos na Central de Regulação, que identificam a emergência e, imediatamente, transferem o telefonema para o médico regulador, que faz o diagnóstico da situação e inicia o atendimento no mesmo instante, orientando a vítima ou a pessoa que fez a chamada, sobre as primeiras ações.

Ao mesmo tempo, o médico regulador em serviço avalia qual o melhor procedimento para a vítima, orienta a pessoa a procurar um posto de saúde; designa uma ambulância de suporte básico de vida, com auxiliar de enfermagem e socorrista para o atendimento no local; ou, de acordo com a gravidade do caso, envia uma unidade avançada de suporte de vida (UTI móvel), com médico e enfermeiro. Com poder de autoridade sanitária, o médico regulador comunica a urgência ou emergência aos hospitais públicos e, dessa maneira, reserva leitos para que o atendimento de urgência tenha continuidade.

\section{O Atendimento Pré-Hospitalar}

Tem por característica atender a vítima nos primeiros minutos após o agravo, de maneira a prestar atendimento adequado e transporte rápido para um estabelecimento de referência ${ }^{(1,18)}$. Tem o objetivo de estabilizar as condições vitais e reduzir a morbimortalidade, por meio de condutas adequadas durante a fase de estabilização e transporte, assim como as iatrogenias que possam culminar com adventos variados, desde as incapacidades físicas temporárias ou permanentes até a morte $\mathrm{e}^{(19)}$.

O APH móvel primário é o socorro oferecido mediante o pedido de um cidadão; o secundário é a solicitação de um serviço de saúde, no qual o usuário está recebendo um primeiro atendimento e necessita ser conduzido a um serviço de maior complexidade ${ }^{(1)}$.

No contexto do APH, as ações são divididas em suporte básico (SBV) e suporte avançado de vida (SAV). O SBV é a estrutura de apoio oferecida a vítimas com risco de morte desconhecido por profissionais de saúde, por meio de medidas conservadoras não-invasivas, tais como: imobilização cervical, contenção de sangramento, curativo oclusivo e imobilização em prancha longa. Inclui ainda ações que visam a qualidade da circulação e oxigenação tecidual, aumentando a chance de sobrevida. O SAV corresponde à estrutura de apoio oferecida por profissionais médicos onde há risco de morte, por intermédio de medidas não invasivas ou invasivas, tais como: intubação endotraqueal, toracocentese, drenagem torácica, pericardiocentese etc. $^{(8,20)}$.

Os serviços de atendimento pré-hospitalar móvel devem contar com equipe de profissionais da área da saúde e outros. Considerando-se que as urgências não se constituem em especialidade médica ou de enfermagem e nos cursos de graduação a atenção dada à área ainda é insuficiente, entende-se que os profissionais que atuam nos Serviços de APH móvel (oriundos e não oriundos da área de saúde), devem ser habilitados pelos Núcleos de Educação em Urgências ${ }^{(1)}$.
Esse serviço conta com o profissional médico regulador, que tem a função de fazer a triagem das chamadas telefônicas e determinar o tipo de suporte mais adequado àquele evento; também pode, dependendo da situação em que se encontra a vítima, se deslocar ao local da emergência ${ }^{(1,3)}$.

Estes serviços visam reduzir o número de óbitos, o tempo de internação em hospitais e as sequelas decorrentes da falta de socorro precoce. Funcionam 24 horas por dia, com equipes de profissionais de saúde, incluindo médicos, enfermeiros, auxiliares e técnicos de enfermagem e socorristas. São atendidas urgências de natureza traumática, clínica, cirúrgica, gineco-obstétrica, problemas mentais e cardiorrespiratórios, bem como intoxicações, queimaduras, quadros infecciosos agudos, maus tratos, tentativas de suicídio e transferência dos usuários entre estabelecimentos de saúde ${ }^{(17)}$.

A Portaria GM №.2048 de 5 de novembro de 2002 define que a equipe de profissionais oriundos da saúde seja composta por: Coordenador do Serviço (profissional da área da saúde, com experiência e conhecimento comprovados na atividade de atendimento pré-hospitalar às urgências e de gerenciamento de serviços e sistemas); Responsável Técnico (responsável pelas atividades médicas do serviço); Responsável de Enfermagem (enfermeiro responsável pelas atividades de enfermagem); Médicos Reguladores (são os responsáveis pelo gerenciamento, definição e operacionalização dos meios disponíveis e necessários para responder às situações informadas pelos usuários, utilizando-se de protocolos técnicos e da escolha sobre os equipamentos de saúde do sistema, necessários ao atendimento); Médicos Intervencionistas (responsáveis pela reanimação e/ou estabilização do usuário, no local do evento e durante o transporte); Enfermeiros Assistenciais (responsáveis pelo atendimento de enfermagem na reanimação e/ou estabilização do paciente, no local do evento e durante o transporte); Auxiliares e Técnicos de Enfermagem (atuam sob supervisão imediata do enfermeiro) $)^{(1)}$.

Essa mesma Portaria dispõe ainda sobre o regulamento técnico dos sistemas estaduais de urgência e emergência, estabelecendo os princípios e diretrizes, as normas e os critérios de funcionamento, a classificação e o cadastramento destes serviços.

O APH móvel é feito em veículos do tipo ambulância (terrestre, aéreo ou aquaviário), destinado exclusivamente ao transporte de enfermos. Suas dimensões e especificações obedecem às normas da ABNT - NBR 14561/2000, de julho de 2000.

As ambulâncias são classificadas em:

- TIPO A: ambulância de transporte - veículo destinado ao transporte em decúbito horizontal de vítimas que não apresentam risco de morte, para remoções simples e de caráter eletivo;

- TIPO B: ambulância de Suporte Básico - veículo destinado ao transporte inter-hospitalar de usuários com risco de morte conhecido e ao atendimento pré-hospitalar de vítimas com risco de morte desconhecido, não classificado com potencial de necessidade de intervenção médica no local e/ou durante o transporte até o serviço de destino;

- TIPO C: ambulância de resgate - veículo de atendimento de urgências pré-hospitalares de vítimas de acidentes ou em locais de difícil acesso, 
com equipamentos de salvamento (terrestre, aquático e em alturas);

- $\quad$ TIPO D: ambulância de Suporte Avançado - veículo destinado ao atendimento e transporte de vítimas de alto risco em emergências pré-hospitalares e/ou de transporte inter-hospitalar que necessitam de cuidados médicos intensivos. Deve contar com equipamentos necessários para esta função;

- TIPO E: aeronave de transporte médico - aeronave de asa fixa ou rotativa utilizada para transporte inter-hospitalar de pacientes e aeronave de asa rotativa para ações de resgate, dotada de equipamentos médicos homologados pelo Departamento de Aviação Civil - DAC;

- TIPO F: embarcação de transporte médico - veículo motorizado aquaviário, destinado ao transporte por via marítima ou fluvial. Deve possuir equipamentos necessários ao atendimento conforme a gravidade dos usuários.

Vários fatores têm contribuído para o aumento da demanda do atendimento pré-hospitalar: o acréscimo do número de acidentes e a violência urbana; a necessidade de aprofundar o processo de consolidação dos Sistemas Estaduais de Urgência e Emergência; a grande extensão territorial do país, que impõe distâncias entre municípios de pequeno e médio porte e seus respectivos municípios de referência para a atenção especializada e de alta complexidade; a necessidade de ordenar o atendimento das urgências e emergências, garantindo acolhimento, atenção qualificada e resolutiva para as pequenas e médias urgências, estabilização e referência adequada dos pacientes graves dentro do Sistema Único de Saúde; a expansão de serviços públicos e privados de APH móvel e de transporte inter-hospitalar e a necessidade de integrar estes serviços à lógica dos sistemas de urgência, com regulação médica e presença de equipe de saúde qualificada para as especificidades deste atendimento ${ }^{(1)}$.

\section{CONSI DERAÇÕES FI NAIS}

O serviço de APH desempenha importante papel na saúde pública, uma vez que as doenças cardiovasculares e os eventos relacionados às causas externas como situações emergenciais são observados cada vez mais na população em geral.

Conhecer a história da implantação dos serviços de atendimento pré-hospitalar tornou evidente que a estruturação de um serviço eficiente e eficaz traz à população segurança e garantia da qualidade na assistência.

Ainda há muito a melhorar, principalmente no que diz respeito à distribuição destes serviços e à continuidade do atendimento nos hospitais de referência. Atualmente a centralização e a disponibilidade limitada de ambulâncias e profissionais estão aquém das necessidades. Por outro lado, a população necessita de mais esclarecimentos dos objetivos do serviço, para sua correta utilização.

Participar do processo de melhoria e do reconhecimento das reais necessidades, nos torna, enquanto profissionais da área da saúde e/ou do APH, coresponsáveis para o alcance do objetivo primordial que é a excelência no atendimento.

\section{REFERÊNCI AS}

1. Ministério da Saúde. Portaria № 2048/GM de 5 de novembro de 2002. Regulamenta o atendimento das urgências e emergências [Internet]. Brasília (Brasil):
Ministério da Saúde; 2002 [cited 2010 set 20]. Available from:

http://dtr2001.saude.gov.br/sas/PORTARIAS/Port2002/Gm/ GM-2048.htm.

2. DATASUS [Internet]. Brasília: Ministério da Saúde (BR) [cited 2010 set 20]. Departamento de Informática do SUS DATASUS. Available

from:

http://www2.datasus.gov. br/DATASUS/index.php.

3. Martins PPS, Prado ML. Enfermagem e serviço préhospitalar: descaminhos e perspectivas. Rev Bras Enferm. 2003; 56(1): 71-5.

4. Minayo MCS, Deslandes SF. Análise da implantação do sistema de atendimento pré-hospitalar móvel em cinco capitais brasileiras. Cad Saude Publica. 2008;24(8):187786.

5. Timerman S, Gonzalez MMC, Mesquita ET, Marques FRB, Ramires JAF, Quilici AP, et al. Aliança Internacional dos Comitês de Ressuscitação (ILCOR). Papel das novas diretrizes de ressuscitação cardiopulmonar e cuidados cardiopulmonares de emergência 2005-2010. Arq Bras Cardiol. 2006; 87(5): e201-8.

6. Silva EAC. Risco biológico para os trabalhadores do Atendimento Pré-Hospitalar [dissertation]. Goiânia: Faculdade de Enfermagem/UFG; 2007.

7. Bíblia. Bíblia sagrada. Trad. José de Almeida. São Paulo: Sociedade Bíblica do Brasil; 1969.

8. Timerman S, Gonzáles MMC, Ramires JAF. Ressuscitação e emergências cardiovasculares. Barueri: Manole; 2007.

9. National Association of Emergency Medical Technicians. PHTLS. Atendimento pré-hospitalar ao traumatizado: básico e avançado. Rio de Janeiro: Elsevier; 2004.

10. Ferrari D. História da ambulância. Revista Intensiva. 2006; 4: 132

11. Nasi LA. Rotinas em pronto-socorro: politraumatizados e emergências ambulatoriais. Porto Alegre: Artes Médicas; 1994.

12. Fernandes RJ. Caracterização da atenção pré-hospitalar móvel da Secretaria da Saúde do município de Ribeirão Preto-SP [dissertation]. Ribeirão Preto: Escola de Enfermagem de Ribeirão Preto/USP; 2004.

13. Ferreira CSW. Os serviços de assistência às emergências no município de São Paulo: implantação de um sistema de atendimento pré-hospitalar [dissertation]. São Paulo: Faculdade de Medicina/Universidade de São Paulo; 1999.

14. Martinez-Almoyna $M$, Nitschke CAS. Regulação médica dos serviços de atendimento médico de urgência - SAMU [Internet]. Florianópolis: Secretaria de Estado da Saúde de Santa Catarina; 1999 [cited 2010 sep 29]. Available from: http://www.saude.sc.gov.br/geral/orgaos_vinculados/samu/ Manual\%20de\%20Regula\% C3\%A7\% C3\% A30\% 20M\%C3\%A 9dica\% 20de\%20Urg\% C3\%AAncia.pdf.

15. Secretaria de Estado de Saúde de Santa Catarina. SAMU 192 [Internet]. Florianópolis: Escola de Saúde Pública. Núcleo de Estudos em Urgências; 2006 [cited 2010 sep 29]. Available from: http://www.scribd.com/doc/14197509/Apostila-Do-Samu-

Santa-Catarina.

16. Timerman S. Suporte básico e avançado de vida em emergências. Brasília: Coordenação de Publicações; 2000.

17. Corpo de Bombeiros Militar do Estado de Goiás [Internet]. Goiânia: Corpo de Bombeiros (BR) [cited 2010 sep 29]. Histórico Geral do Corpo de Bombeiros Militar do Estado de Goiás. Available from: http://www. bombeiros.go.gov.br/historico/. 
18. Lopes ACS, Oliveira AC, Silva JT, Paiva MHRS. Adesão às precauções padrão pela equipe do atendimento préhospitalar móvel de Belo Horizonte, Minas Gerais, Brasil. Cad Saude Publica. 2008;24(6): 1387-96.

19. Pavalqueires S. Educação continuada de enfermeiros no atendimento inicial à vítimas de traumatismos [dissertation]. Ribeirão Preto: Escola de Enfermagem de Ribeirão Preto/USP; 1997.

20. American Heart Association Guidelines for Cardiopulmonary Resuscitation and Emergency Cardiovascular Care. Circulation. 2005;112(24 Suppl):IV1203.

Artigo recebido em 20.04.2009

Aprovado para publicação em 19.05.2010

Artigo publicado em 30.09.2010 